\title{
Key Role of Mucosal Primary Afferents in Mediating the Inhibitory Influence of Capsaicin on Vagally Mediated Contractions in the Mouse Esophagus
}

\author{
Ammar BOUDAKA ${ }^{1)}$, Jürgen WÖRL ${ }^{1,3)}$, Takahiko SHIINA ${ }^{1)}$, Shouichiro SAITO2), Yasuro ATOJI ${ }^{2}$, \\ Haruo KOBAYASHI ${ }^{1)}$, Yasutake SHIMIZU ${ }^{1) *}$ and Tadashi TAKEWAKI ${ }^{1)}$ \\ ${ }^{1)}$ Laboratory of Physiology, Department of Basic Veterinary Science and ${ }^{2)}$ Laboratory of Anatomy, Department of Basic Veterinary \\ Science, The United Graduate School, Gifu University, Yanagido 1-1, Gifu 501-1193, Japan and ${ }^{3)}$ Institute of Anatomy, University of \\ Erlangen-Nuremberg, Krankenhausstrasse 9, D-91054 Erlangen, Germany
}

(Received 4 October 2006/Accepted 4 December 2006)

\begin{abstract}
Transient receptor potential ion channel of the vanilloid type 1 (TRPV1)-dependent pathway, consisting of capsaicin-sensitive tachykininergic primary afferent and myenteric nitrergic neurons, was suggested to mediate the inhibitory effect of capsaicin on the vagally mediated striated muscle contractions in the rat esophagus. These primary afferent neurons upon entering into the esophagus are distributed through the myenteric plexus, terminating either in the myenteric ganglia or en route to the mucosa where they branch into a delicate net of fine varicose fibers. Therefore, this study aimed to investigate whether the mucosal primary afferents are a main mediator for the capsaicin inhibitory influence on vagally mediated contractions in the mouse esophagus. For this purpose, the vagally induced contractile activity of a thoracic esophageal segment was measured in the circular direction with a force transducer. Vagal stimulation $(30 \mu \mathrm{sec}, 25 \mathrm{~V}, 1-50 \mathrm{~Hz}$ for $1 \mathrm{sec})$ produced monophasic contractile responses, whose amplitudes were frequency-dependent. These contractions were completely abolished by d-tubocurarine $(5 \mu \mathrm{M})$ while resistant to atropine $(1 \mu \mathrm{M})$ and hexamethonium $(100$ $\mu \mathrm{M})$. Capsaicin $(30 \mu \mathrm{M})$ significantly inhibited the vagally induced contractions in esophagi with intact mucosa while its effect on preparations without mucosa was insignificant. Additionally, immunocytochemistry revealed the presence of TRPV1-positive nerve fibers in the tunica mucosa. Taken together, we conclude that in the mouse esophagus, capsaicin inhibits the vagally mediated striated muscle contractions mainly through its action on mucosal primary afferents, which in turn activate the presumed inhibitory local reflex arc. KEY WORDS: capsaicin-sensitive neurons, enteric co-innervation, local effector function, TRPV1, vagus nerve.
\end{abstract}

A peripheral reflex arc composed of capsaicin-sensitive tachykininergic primary afferent and myenteric nitrergic neurons have been shown to negatively influence the vagally mediated striated muscle contractions in the rat esophagus [27]. Esophageal neuromuscular junctions receive a dual innervation from both vagal nerve fibers originating in the brain stem and from varicose enteric nerve fibers originating in the myenteric plexus, the so-called enteric co-innervation [for review see 21,34]. Enteric neurons in the esophagus are contacted by primary afferents of spinal and vagal origin [19, 20, 25]. A substantial number of these primary afferents, mainly of spinal origin, were shown to be immunoreactive to the transient receptor potential ion channel of the vanilloid type 1 (TRPV1) and called capsaicin-sensitive sensory neurons $[12,14,15,23,30,31$, 33]. These neurons can modulate intestinal motility by transferring signals from the gastrointestinal tract to the central nervous system and simultaneously releasing transmitters, in particular substance P (SP) and calcitonin generelated peptide (CGRP) that can influence the activity of intrinsic neurons $[14,16]$.

Primary afferents, of spinal and vagal origins, terminate in the esophagus either on myenteric ganglia where they function mainly as mechanosensors, or en route to the mucosa where they branch into a delicate net of fine vari-

\footnotetext{
* Correspondence to: Shimizu, Y., Laboratory of Physiology, Department of Basic Veterinary Science, The United Graduate School, Gifu University, 1-1 Yanagido, Gifu 501-1193, Japan.
}

cose fibers. Some of the mucosal afferents have intraepithelial extensions suggesting thermo- or chemosensory functions [22]. So far, it is still unknown whether mucosal primary afferents play a key role in mediating capsaicin inhibitory effect on the vagally induced contractions of esophageal striated muscle fibers as part of a local reflex arc that was previously suggested by our group [17, 27].

Capsaicin is the pungent ingredient in a wide variety of red peppers of the genus Capsicum and acts on afferent neurons through activation of specific receptors, first named as capsaicin receptor or vanilloid receptor of type 1 (VR1) [4, 5] and later renamed as TRPV1 [6, 7]. Capsaicin-sensitive sensory neurons can modulate intestinal motility by transferring signals from the gastrointestinal tract to the central nervous system and simultaneously releasing transmitters that can influence the activity of intrinsic neurons, the socalled local effector function [13, 16, 29]. The action of capsaicin on afferent neurons involves two phases: an acute excitatory effect which leads to transmitter release, followed by desensitization, i.e. the neurons become unresponsive to further applications of the drug [14].

The main aim of this study was to investigate whether the inhibitory effect of capsaicin on vagally mediated contractions is dependent on the mucosal primary afferents. For this purpose, we used in vitro vagus nerve-esophagus preparations where mucosa was kept intact in some preparations, while it was surgically removed in other preparations. Successful removal of the mucosal layer was confirmed by 
hematoxylin and eosin staining at the end of each experiment. Additionally, the presence of TRPV1-positive nerve fibers was checked by immunocytochemistry. Our results suggest that capsaicin-sensitive mucosal primary afferents have a major role in mediating the inhibitory effect of capsaicin on vagally induced contractions in the mouse esophagus.

\section{MATERIALS AND METHODS}

Animals and tissue preparation: Forty-seven ddY mice of both sexes (9-13 weeks old, body weights 27-31 g for females and 39-45 g for males) were obtained from Japan SLC (Shizuoka, Japan). They were maintained in acrylic cages at $22 \pm 2{ }^{\circ} \mathrm{C}$ with a 12-hr light/dark cycle (light on 07:00-19:00 hr) and with free access to water and laboratory chow (LABO MR Stock, Nihon-Nosan, Yokohama, Japan). The experiments were performed in accordance with the Gifu University Animal Care and Use Committee guidelines on animal care and use. The number of animals used was minimized. For in vitro experiments, animals were anaesthetized with sodium pentobarbital $(100 \mathrm{mg} / \mathrm{kg}$ i.p.) and exsanguinated via the axillary artery. The abdominal and thoracic cavities were opened immediately. Then the stomach, esophagus up to the larynx with attached vagus nerves, heart, and lungs were quickly removed and placed in a dish containing precooled aerated physiological salt solution (PSS, see below). A 2-3 cm-long segment of the esophagus with both vagus nerves was carefully dissected out of the mediastinal tissue under a light microscope with great care not to damage the vagus nerves. Finally, a thoracic segment of 5-7 $\mathrm{mm}$ in length distal to the bifurcation of the trachea was obtained for mechanical recordings. In some preparations, the mucosa was surgically removed by blunt dissection under light microscope visualization. For TRPV1-immunocytochemistry, mice were euthanized at the age of 3 months with an overdose of sodium pentobarbital $(250 \mathrm{mg} / \mathrm{kg}$ i.p.) and the thoracic esophageal segments were dissected and fixed by immersion in $4 \%$ phosphate-buffered formaldehyde, $\mathrm{pH} 7.4$ for $7 \mathrm{hr}$ at $4^{\circ} \mathrm{C}$. After rinsing in $12 \%$ buffered sucrose for cryoprotection overnight, the tissue segments were rapidly frozen in isopentane cooled in liquid nitrogen.

Mechanical recordings: The dissected segment was transferred to a thermostatically controlled $\left(35^{\circ} \mathrm{C}\right)$ organ bath of $5 \mathrm{ml}$ in capacity filled with PSS and continuously bubbled with a $95 \% \mathrm{O}_{2}+5 \% \mathrm{CO}_{2}$ gas mixture. Two fine Lshaped stainless-steel pins, each of $150 \mu \mathrm{m}$ in diameter, were introduced through the lumen of the esophageal segment. One pin was fixed to the bottom of the organ bath and the other was connected to the bar of an isometric force transducer (T7-30-240, Orientec, Tokyo, Japan). Contractile activity was measured in the circular direction. An initial resting tension of $0.5 \mathrm{~g}$ was applied to the esophageal preparations, which were subsequently allowed to equilibrate for 30-45 min. At the end of this period, the tension created by the esophageal segment was considered as the resting tension and no further mechanical adjustment was made during experimentation. Isometric responses were filtered and amplified by an amplifier (NEC, AS1202, Tokyo, Japan) and recorded using a PowerLab system (AD Instruments, Bella Vista NSW, Australia).

Vagal stimulation: In experiments using vagal stimulation, the end of the vagus nerve trunk was drawn into a bipolar suction electrode placed $10 \mathrm{~mm}$ from the esophagus and the electrode was immersed together with the esophagus preparation in the bath. The distance of $10 \mathrm{~mm}$ between the esophagus and stimulating suction electrode excludes direct muscular activation, since electrical stimulation at this distance did not cause a contractile response in preparations lacking neuronal connection. The vagus nerve was stimulated using an electronic stimulator (model SEN-3301, Nihon Kohden, Tokyo, Japan) connected to the suction electrode. Single square-wave pulses of $30 \mu \mathrm{sec}$ in duration and $25 \mathrm{~V}$ in intensity were applied periodically at 1-min intervals. To test the effects of different drugs, each protocol was started with single pulses of vagal stimulation applied periodically for 15-20 min under standard conditions and used as a control. Then the respective agonist or antagonist was added to the organ bath in microliter volumes and periodical vagal stimulation was continued as described above for $30 \mathrm{~min}$. This drug application period was followed by repeated washout with PSS for $3 \mathrm{~min}$, and then the tissue was allowed to rest for 30 min before any subsequent drug application.

For train stimulation, square-wave pulses $(30 \mu \mathrm{sec}$ in duration and $25 \mathrm{~V}$ in intensity) of variable frequencies (1-50 $\mathrm{Hz}$ ) were applied periodically for $1 \mathrm{sec}$ at 5-min interval. Every in vitro experiment lasted less than $4 \mathrm{hr}$ and each oesophagus was used for a single experiment only.

Electrical field stimulation: Electrical field stimulation was also applied through a pair of platinum electrodes, each placed on one side of the tissue. For the stimulation of the muscle tissue, single square-wave pulses of $10 \mathrm{msec}$ in duration and $25 \mathrm{~V}$ in intensity were delivered at 1-min intervals using an electronic stimulator (model SEN-3301, Nihon Kohden, Tokyo, Japan) connected to the electrodes. Single pulses were delivered for 15-20 min and used as a control, and then the test drug was applied for $30 \mathrm{~min}$ followed by repeated washout with PSS for $3 \mathrm{~min}$, and the tissue was allowed to rest for $30 \mathrm{~min}$ before any further experimentation. Transmural stimulation was employed to check any direct muscular action of the tested drugs, at the used concentrations, on basal tension and twitch contractions.

Physiological salt solution and drugs: The PSS used in this study had the following composition in $\mathrm{mM}: \mathrm{NaCl}$ 136.9, $\mathrm{KCl} 2.7, \mathrm{CaCl}_{2}$ 1.8, $\mathrm{MgCl}_{2} 2.1, \mathrm{NaH}_{2} \mathrm{PO}_{4} 0.4$, $\mathrm{NaHCO}_{3} 11.9$ and glucose 5.5. The solution in the organ bath was gassed continuously with a $95 \% \mathrm{O}_{2}+5 \% \mathrm{CO}_{2}$ gas mixture creating a $\mathrm{pH}$ of 7.4 and was maintained at $35^{\circ} \mathrm{C}$.

Atropine, d-tubocurarine, hexamethonium bromide, tetrodotoxin and capsaicin were obtained from Sigma Chemical Co. (St Louis, MO, U.S.A.). Capsaicin was dissolved in ethanol. Other drugs were dissolved in distilled water. The 
concentrations of stock solutions were higher by 100 times or more than those of solutions used for the experiments, and further dilutions were made in PSS. Final concentration of ethanol (solvent used to prepare capsaicin) in the bathing solution was $0.03 \%(\mathrm{v} / \mathrm{v})$ and had no effect on the tone of the tissues or on the amplitude of vagally induced contractions when tested separately. The drug concentrations given in the text are the final concentrations in the bathing solution. Unless stated otherwise, capsaicin was added only once to each esophageal preparation due to its desensitization effect on capsaicin-sensitive neurons.

Hematoxylin and eosin staining: At the end of each functional experiment, the esophageal preparation was fixed by immersion in $4 \%$ phosphate-buffered formaldehyde, $\mathrm{pH} 7.4$, for $7 \mathrm{hr}$ at $4^{\circ} \mathrm{C}$. The fixed tissues were embedded in paraffin using standard methods, sectioned longitudinally, and mounted on glass slides. The sections were stained with hematoxylin and eosin, dehydrated in ethanol series, cleared with xylene, sealed with cover slips and examined under a conventional light microscope to prove the presence or absence of the mucosa. Images were captured with a digital camera (Pro 600ES, Pixera Corporation, Los Gatos, CA, U.S.A.) mounted on the microscope and adjusted for brightness and contrast in Adobe Photoshop 6.0 (Adobe Systems, San Jose, U.S.A.).

Immunocytochemistry: Longitudinal sections of the lower thoracic portion of the esophagus were taken at $14 \mu \mathrm{m}$ on a Microm cryostat (Microm GmbH, Heidelberg, Germany), mounted on poly-L-lysine-coated slides, air-dried for $1 \mathrm{hr}$, and preincubated with $1 \%$ bovine serum albumin (BSA), $0.5 \%$ Triton X-100 and 10\% normal donkey serum for $1 \mathrm{hr}$ at room temperature. After being rinsed in TRIS-buffered saline (TBS; pH 7.4), sections were incubated in a humid chamber with a primary rabbit antibody against a synthetic peptide corresponding to amino acid residues $824-838$ of the rat capsaicin receptor (later renamed to TRPV1; Calbiochem, Darmstadt, Germany) diluted in TBS (1:500), containing $1 \%$ BSA and $0.5 \%$ Triton $\mathrm{X}-100$ at room temperature overnight. Following three washes with TBS, antibody-binding sites were visualized by Alexa-488-conjugated donkey anti-rabbit IgG (Molecular Probes; Eugene, USA) diluted in TBS $(1: 1,000)$ for $1 \mathrm{hr}$ at room temperature. The sections were rinsed and cover slipped in TBS-glycerol (1:1, pH 8.6). For conventional fluorescence microsocopy a Leica Aristoplan microscope fitted with appropriate filter settings for viewing Alexa-488 fluorescence was used. Black/white images were captured via a charge-coupled device camera (Visitron Systems, Puchheim, Germany) and adjusted for brightness and contrast in Adobe Photoshop 6.0 (Adobe Systems, San Jose, U.S.A.).

Data processing and statistical analysis: The number of separate esophageal preparations in which mechanical responses were recorded is indicated by (n). For frequency response analysis, the mean of the twitch contraction magnitude for 5 preparations at each frequency in the range of 1$50 \mathrm{~Hz}$ was expressed as percentage of the maximum obtained response. For the drug effect, the mean of three consecutive twitches was expressed as percent change compared to the control value prior to drug application. Control experiments using the above-described protocols for vagal stimulation and electrical field stimulation showed no significant changes in striated muscle responses over a period of $4 \mathrm{hr}$.

Data are presented as means and standard error of the mean (S.E.M.). The significance of differences between the means was determined by ANOVA, with post-hoc testing using Duncan's multiple-range test as appropriate. P values $\leq 0.05$ were considered significant.

\section{RESULTS}

Pharmacological characterization of vagal stimulationinduced and electrical field stimulation-induced contractile responses in the mouse esophagus: No spontaneous mechanical activity was recorded at rest from the esophageal segment used in the present in vitro investigation. The resting tension recorded at the end of the equilibration period of $30-45 \mathrm{~min}$ was $0.23 \pm 0.02 \mathrm{~g}(\mathrm{n}=21)$. Vagal stimulation $(30 \mu \mathrm{sec}, 25 \mathrm{~V})$ produced monophasic contractile responses that were stable in magnitude for over $4 \mathrm{hr}$. These single components of the contractile responses were frequency-dependent (Fig. 1A). Frequency-response relationship was determined for vagal stimulation from $1-50 \mathrm{~Hz}$ (Fig. 1B). No second component [1, 18, 28] was detected after the first component at all frequencies. For further experiments, we used single pulses delivered at 1-min interval, since the twitch contractions were stable in magnitude for longer time with single stimulation protocol.

d-Tubocurarine $(5 \mu \mathrm{M})$, a blocker of the nicotinic acetylcholine receptors of the motor endplate, almost completely inhibited the vagally induced contractions (Fig. 2A). Atropine $(1 \mu \mathrm{M})$ and hexamethonium $(100 \mu \mathrm{M})$, blockers of muscarinic acetylcholine receptors and ganglionic nicotinic acetylcholine receptors, respectively, did not affect the twitch responses to vagal stimulation (Fig. 2A). These data indicated that the vagally induced twitch contractile responses were mediated by acetylcholine via nicotinic acetylcholine receptors on the motor endplates. Tetrodotoxin, a blocker of the neuronal voltage-dependent sodium channels, significantly inhibited the twitch responses to vagal stimulation (Fig. 2A).

Electrical field stimulation (single pulses, $10 \mathrm{msec}, 25 \mathrm{~V}$ every $1 \mathrm{~min}$ ) of the esophageal segment produced monophasic contractile responses that were stable in magnitude for over $4 \mathrm{hr}$. The contractile responses were unaffected by dtubocurarine $(5 \mu \mathrm{M})$, atropine $(1 \mu \mathrm{M})$ and hexamethonium $(100 \mu \mathrm{M})$ but were significantly inhibited by tetrodotoxin in a concentration-dependent manner (Fig. 2B).

At the concentrations used, none of the tested drugs (dtubocurarine, atropine, hexamethonium and tetrodotoxin) per se had any significant effect on the resting tension of the esophageal preparations.

Vagal stimulation of in vitro vagus nerve-esophagus preparations lacking mucosa produced monophasic contrac- 
A
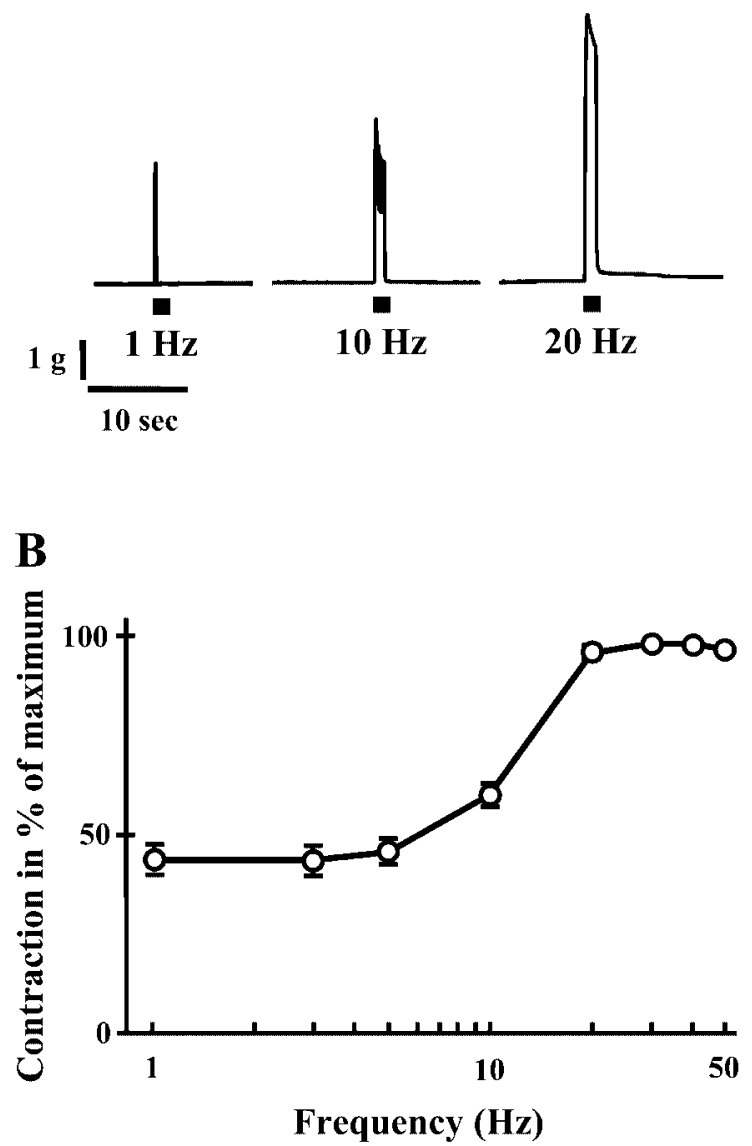

Fig. 1. Contraction of esophageal striated muscles in response to vagal stimulation at different frequencies. (A) Original tracings; (B) frequency-response curves (maximal contractions $=100 \%)$. Vagal stimulation was delivered at standard parameters of $25 \mathrm{~V}, 30 \mu \mathrm{sec}$ pulse duration, $1 \mathrm{sec}$ stimulation duration at frequencies ranging from $1-50 \mathrm{~Hz}$. Each data point represents the mean \pm SEM of five experiments.

tile responses of amplitudes comparable to preparations with intact mucosa. Likewise, d-tubocurarine $(5 \mu \mathrm{M})$ almost completely inhibited the vagally induced contractions in these preparations ( $\mathrm{n}=5$, data not shown).

Effects of capsaicin on vagal stimulation-induced and electrical field stimulation-induced contractile responses in mouse esophagus with intact mucosa: Capsaicin $(30 \mu \mathrm{M})$ transiently inhibited the vagally induced contractions in the mouse esophagus and the twitches recovered to the control level in the presence of the drug (Fig. 3A). Following a period of washout with PSS and resting, subsequent applications of capsaicin $(30 \mu \mathrm{M})$ failed to inhibit the vagally mediated twitch contractions ( $\mathrm{n}=5$, data not shown). This can be attributed to the desensitization phenomenon exhibited by the TRPV1. Capsaicin, at the above used concentration, had no effect on electrical field stimulation-induced response
(Figs. 3A) or resting tension.

Effects of capsaicin on vagal stimulation-induced and electrical field stimulation-induced contractile responses in mouse esophagus lacking mucosa: Capsaicin (30 $\mu \mathrm{M})$ significantly lost its inhibitory effect on vagally induced contractions in esophageal preparations lacking mucosal layer (Figs. 3B and 4). Capsaicin, at the above used concentration, had no effect on electrical field stimulation-induced response (Figs. 3B) or resting tension of these preparations.

Confirmation of mucosa removal by hematoxylin and eosin staining: Hematoxylin and eosin staining of longitudinal sections obtained from the thoracic esophagus with intact mucosa revealed that the tunica muscularis was composed of multinucleated striated muscle fibers. The lamina epithelialis mucosae was composed of stratified squamous epithelium, while the lamina muscularis mucosae consisted of smooth muscle bundles (Fig. 5A). On the other hand, staining of preparations whose mucosal layer was removed by blunt dissection revealed that the tunica mucosa with its lamina muscularis mucosae was torn away from the underlying tunica muscularis at the level of tela submucosa (Fig. 5B).

Presence of TRPV1-positive nerve fibers in the tunica mucosa of the mouse esophagus: Immunocytochemical labeling for TRPV1 revealed the presence of TRPV1-positive fine varicose nerve fibers in the tunica mucosa, in particular in the lamina propria mucosae in close contact to the epithelium (Fig. 6) and in the tunica muscularis in close contact to myenteric ganglia. TRPV1-immunoreactive nerve cell bodies could be detected occasionally in the myenteric plexus, but never in the tunica mucosa or tela submucosa of the thoracic esophagus.

\section{DISCUSSION}

In the present study, the effect of mucosal layer removal on capsaicin-induced inhibition of the vagally evoked contractions was examined to clarify whether mucosal primary afferents play a key role in mediating the inhibitory effect of capsaicin. The main findings in this study are: 1) mechanical recording from the circular direction of vagally stimulated mouse esophagus resulted in striated muscle mediated contractile responses that were frequency-dependent, 2) in preparations with intact mucosa, capsaicin inhibited the vagally evoked twitch responses but did not affect the electrical field stimulation evoked contractions, 3) mucosa removal significantly attenuated the inhibitory influence of capsaicin on vagally induced twitch contractions and 4) immunocytochemistry revealed the presence of TRPV1positive nerve fibers in the tunica mucosa. These results, together with our recent findings [17, 27], suggest that a TRPV1-dependent local neural reflex, which can be influenced by capsaicin, might regulate the vagally mediated striated muscle motility in mice. Additionally, the results suggest the dependence of capsaicin on mucosal primary afferents for evoking its inhibitory effect.

The tunica muscularis of the mouse esophagus is entirely 
A. VS
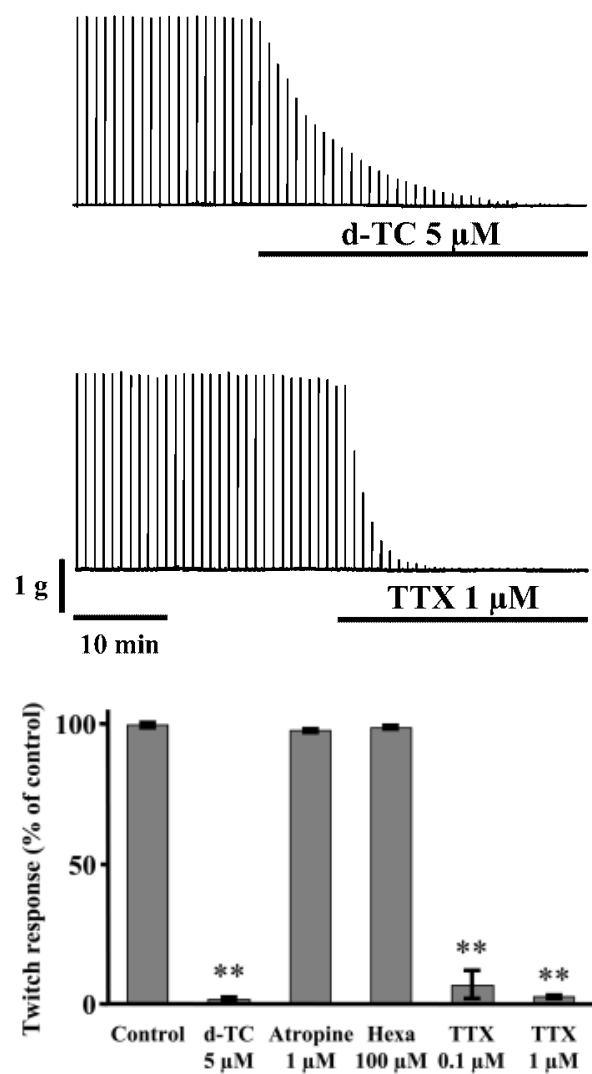

B. EFS
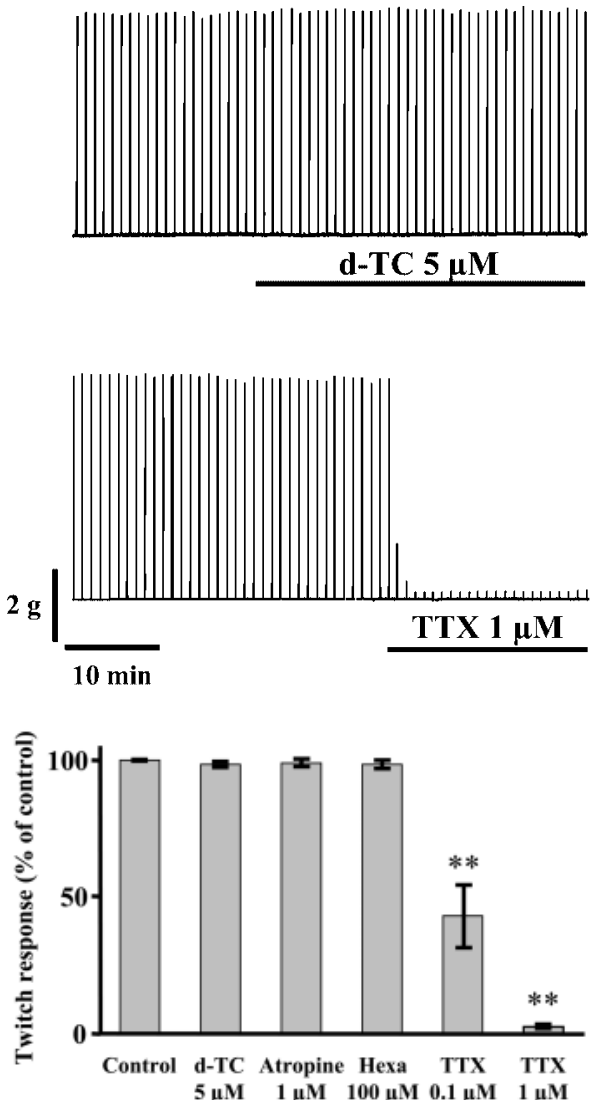

Fig. 2. The influence of different drugs on vagal stimulation (VS)-induced and electrical field stimulation (EFS)-induced contractions in the mouse esophagus. Original traces and bar graphs demonstrating the influence of d-tubocurarine (d-TC; $5 \mu \mathrm{M})$, atropine $(1 \mu \mathrm{M})$, hexamethonium (Hexa; $100 \mu \mathrm{M})$ and tetrodotoxin (TTX; $0.1 \mu \mathrm{M}, 1 \mu \mathrm{M}$ ) on esophageal response to (A) VS (single pulses, $30 \mu \mathrm{sec}$ and $25 \mathrm{~V}$ ) and (B) EFS (single pulses, $10 \mathrm{msec}$ and $25 \mathrm{~V}$ ). Each bar represents the mean \pm SEM of five experiments. $* * P<0.01$, compared with the control that is the response before the addition of the respective drug.

composed of striated muscle fibers arranged into incompletely separated outer longitudinal and inner circular layers [34]. In the present study, we recorded the mechanical activities from the inner muscle layer, because the contraction amplitude of the esophageal peristalsis is mainly a function of circularly arranged muscle fibers [8, 9, 24, 26]. Vagal stimulation of the mouse esophagus elicited fast monophasic contractile responses that were abolished by $\mathrm{d}$ tubocurarine but not by atropine or hexamethonium, indicating the involvement of cholinergic neuromuscular neurotransmission via nicotinic acetylcholine receptors at the motor endplates. The lack of a second slow component from the obtained results by single and multiple pulses at high frequencies, which have been previously described using repetitive vagal stimulation of the guinea pig and rat esophageal preparations in the longitudinal direction $[1,18$, 28 ], is in good agreement with the view that these components were attributed to the contraction of the lamina muscularis mucosae. This later is entirely composed of smooth muscle fibers oriented in a longitudinal direction $[1,11]$. Thus, mechanical recoding from the circular layer of the mouse esophagus is a suitable technique for experiments focusing on the physiological significance of the enteric coinnervation of the esophageal motor endplates.

The primary afferent neurons, of spinal and vagal origins, convey sensory information from the upper gastrointestinal tract to the central nervous system. A substantial number of these primary afferents, mainly of spinal origin, were shown to be immunoreactive to TRPV1 and called capsaicin-sensitive sensory neurons $[12,14,15,23,30,31,33]$. In this study, capsaicin transiently inhibited the vagally mediated esophageal muscle contractions. Capsaicin effect is most probably mediated by its activation of TRPV 1 since it fails to inhibit the vagally evoked contractions in preparations pretreated with capsaicin, which can be explained by desensitization of TRPV1 on the primary afferent neurons [14]. Similar findings were previously obtained in hamsters and rats $[17,27]$. Capsaicin is unlikely to have a direct inhibi- 
A. With mucosa

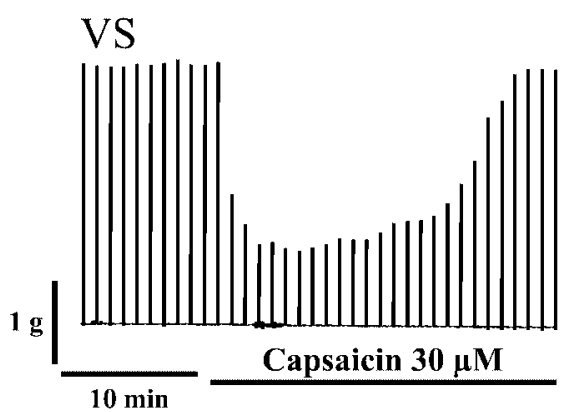

EFS

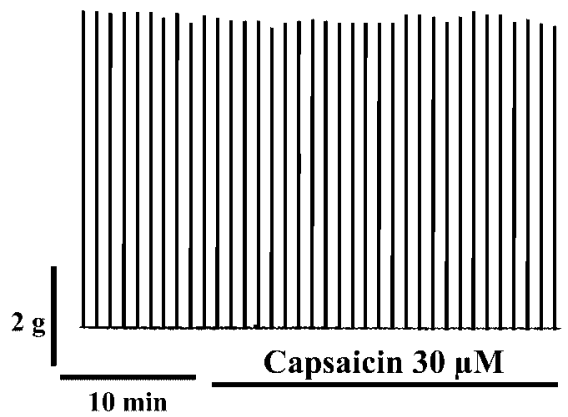

B. Without mucosa

VS

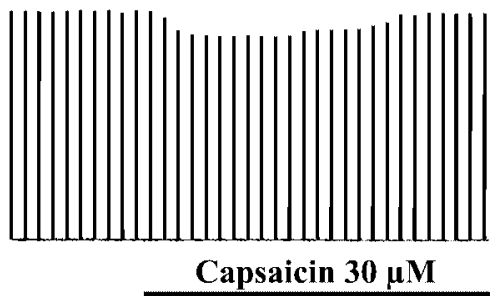

EFS

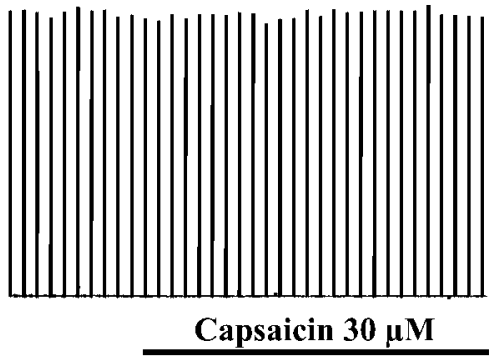

Fig. 3. The effect of capsaicin $(30 \mu \mathrm{M})$ on vagal stimulation (VS)-induced and electrical field stimulation (EFS)-induced twitch contractions in esophagi (A) with and (B) without mucosa. Control recordings of VS (single pulses, $30 \mu \mathrm{sec}$ and $25 \mathrm{~V}$ ) and EFS (single pulses, $10 \mathrm{msec}$ and $25 \mathrm{~V}$ ) evoked contractions for $15 \mathrm{~min}$ were followed by application of capsaicin $(30 \mu \mathrm{M})$.

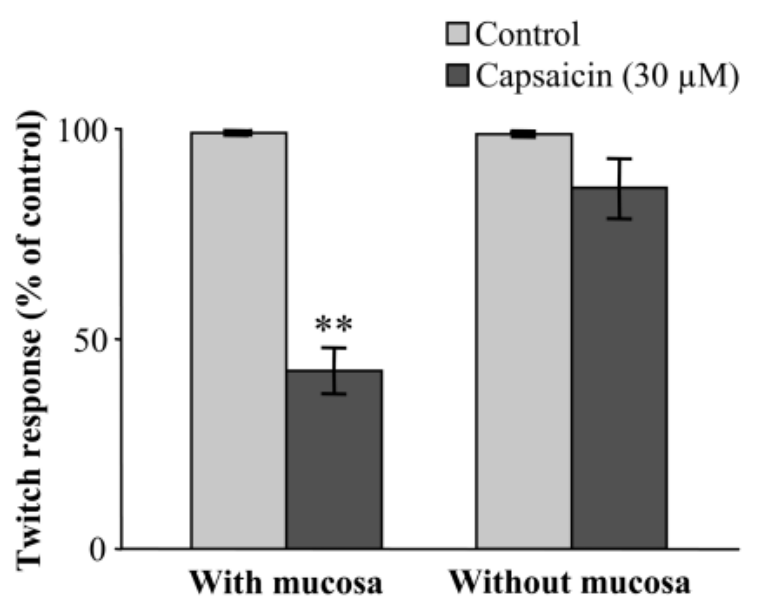

Fig. 4. The effect of mucosa removal on the inhibitory influence of capsaicin on vagally evoked contractions in the mouse esophagus. Vagal stimulation was delivered as single pulses of $30 \mu \mathrm{sec}$ duration and $25 \mathrm{~V}$ intensity. Each bar represents the mean \pm S.E.M. of six experiments. $* * P<0.01$, compared with the control that is the response before the addition of the respective drug. tory effect on the esophageal striated muscles since it has no effect on contractions achieved by electrical field stimulation.

Mucosal layer removal significantly attenuated the response of esophageal preparations to the inhibitory effect of capsaicin. Our method for mucosal layer removal by blunt dissection seems to be successful in eliminating the tunica mucosa since this layer fails to show up on longitudinal sections of such preparations that were stained with hematoxylin and eosin (Fig. 5B). The mucosa of the alimentary tract, in addition to its absorptive and secretive functions, serves as a sensory organ that perceives different sensory modalities and transducts them to the enteric as well as the central nervous systems. Vagal innervation of the mucosa shows the greatest density in the upper cervical esophagus which steeply decreases in the lower cervical and thoracic esophagus $[10,20,32]$. On the other hand, spinal afferents are distributed rather evenly from oral to aboral [10]. Since we have used thoracic esophageal segments in the current study, we can suggest that mucosal capsaicinsensitive primary afferents, mainly of spinal origin, play an important role in mediating the inhibitory influence of capsaicin on vagally mediated contractions. In agreement with this later suggestion, immunocytochemical labeling for TRPV1 clearly demonstrated the presence of TRPV1-posi- 

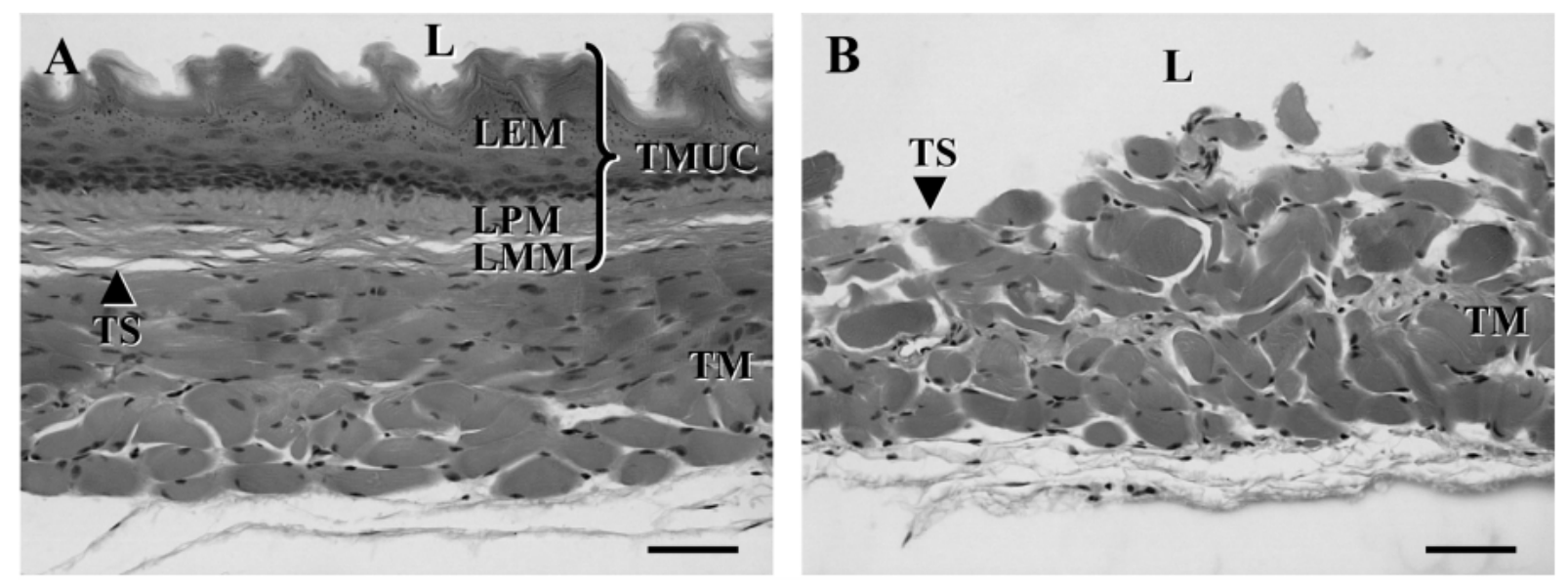

Fig. 5. Thoracic portion of the mouse esophagus stained with hematoxylin and eosin. (A) Section from a preparation with intact mucosa showing all characteristic layers of the esophageal wall. (B) Represents a preparation whose mucosal layer was removed by blunt dissection. We can notice that the tunica mucosa was torn at the level of tela submucosa. Scale bar $=50 \mu \mathrm{M}$. $\mathrm{L}=$ lumen, TMUC=tunica mucosa, LEM=lamina epithelialis mucosae, LPM=lamina propria mucosae, LMM=lamina muscularis mucosae, TS=tela submucosa, $\mathrm{TM}=$ tunica muscularis.

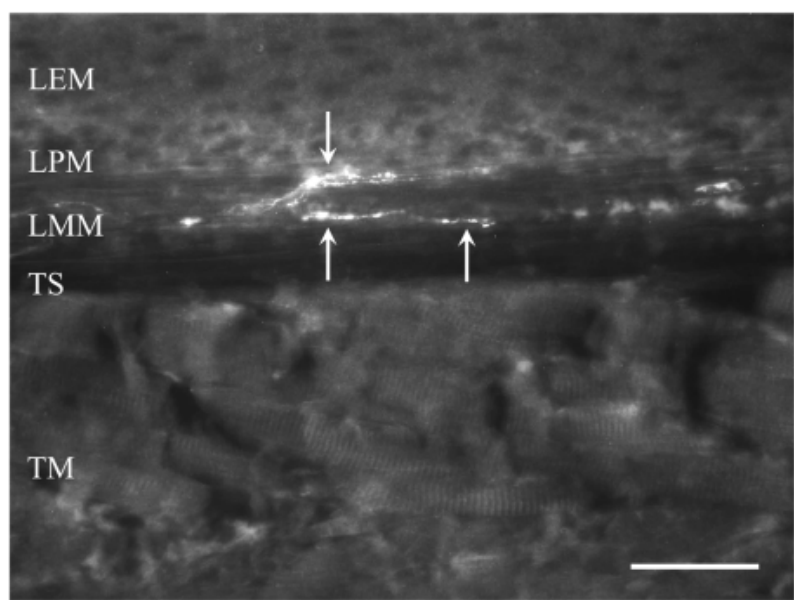

Fig. 6. Conventional fluorescence preparation showing mucosal TRPV1-positive nerve fibers in the mouse esophagus. TRPV1immunoreactive nerve fibers (arrows) in the lamina muscularis and lamina propria mucosae close to the epithelium of a longitudinal section obtained from the thoracic esophagus. Scale bar $=50$ $\mu \mathrm{M}$. LEM=lamina epithelialis mucosae, LPM=lamina propria mucosae, LMM=lamina muscularis mucosae, TS=tela submucosa, $\mathrm{TM}=$ tunica muscularis.

tive fine varicose nerve fibers running in the tunica mucosa, particularly in the lamina propria mucosae in close contact to the epithelium (Fig. 6). The persistence of a weak, although insignificant, effect of capsaicin on the vagally mediated contractions in preparations lacking the mucosal layer (Fig. 3B) could be attributed to the action of capsaicin on TRPV1-immunoreactive nerve cell bodies that were occasionally detected in the myenteric plexus of the mouse esophagus. Consequently, the activated TRPV1-positive myenteric neurons can influence the vagal efferents co- innervated by the enteric fibers arising from those neurons $[21,34]$.

Tetrodotoxin is used as a selective neuronal blocker in many experimental setups. However, in previous reports $[18,27,28]$ and in the present investigation, tetrodotoxin (1 $\mu \mathrm{M})$ also significantly inhibited contractions of the esophageal muscle, which were induced by electrical field stimulation. These electrical field stimulation-induced twitches could not be inhibited by d-tubocurarine, indicating that tetrodotoxin at this concentration not only blocks neural transmission but also directly inhibits the striated muscle contraction. Voltage-dependent sodium channels are distributed with different densities over the entire muscle cell membrane at least in skeletal muscles [2,3] and might reflect the sensitivity of esophageal striated muscle fibers to tetrodotoxin.

In conclusion, this study shows that in the mouse esophagus, capsaicin inhibits the vagally mediated striated muscle contractions mainly through its action on mucosal primary afferents, probably of spinal origin, which in turn activate the presumed inhibitory local reflex arc.

ACKNOWLEDGEMENTS. We thank Prof. Makoto Tominaga (National Institutes of Natural Sciences, Okazaki, Japan) for helpful and stimulating discussions. This work was supported by a travel grant to J.W. from the Chihiro and Kiyoko Yokochi Found, Kanehara Ichiro Foundation in Tokyo, Japan.

\section{REFERENCES}

1. Beveridge, A.A. and Taylor, G.S. 1987. Comparison of nervous control of smooth and skeletal muscle. Proc. Aust. Physiol. Pharmacol. Soc. 18: 203-210.

2. Caldwell, J.H. 2000. Clustering of sodium channels at the neuromuscular junction. Microsc. Res. Tech. 49: 84-89. 
3. Caldwell, J.H. and Milton, R.L. 1988. Sodium channel distribution in normal and denervated rodent and snake skeletal muscle. J. Physiol. (Lond.) 401: 145-161.

4. Caterina, M.J.and Julius, D. 2001. The vanilloid receptor: a molecular gateway to the pain pathway. Ann. Rev. Neurosci. 24: 487-517.

5. Caterina, M.J., Schumacher, M.A., Tominaga, M., Rosen, T.A., Levine, J.D. and Julius, D. 1997. The capsaicin receptor: a heat-activated ion channel in the pain pathway. Nature 389: 816-824.

6. Clapham, D.E., Julius, D., Montell, C. and Schultz, G. 2005. International Union of Pharmacology. XLIX. Nomenclature and structure-function relationships of transient receptor potential channels. Pharmacol. Rev. 57: 427-450.

7. Clapham, D.E., Montell, C., Schultz, G. and Julius, D. 2003. International Union of Pharmacology. XLIII. Compendium of voltage-gated ion channels: transient receptor potential channels. Pharmacol. Rev. 55: 591-596.

8. Conklin, J.L. 1998. Nitric oxide: a mediator of esophageal motor function. J. Lab. Clin. Med. 131: 10-20.

9. Conklin, J.L. and Christensen, J. 1994. Motor function of the pharynx and esophagus. pp. 903-928. In: Physiology of the Gastrointestinal Tract (Johnson, L.R. ed.), Raven Press, New York.

10. Dütsch, M., Eichhorn, U., Wörl, J., Wank, M., Berthoud, H.R. and Neuhuber, W.L. 1998. Vagal and spinal afferent innervation of the rat esophagus: a combined retrograde tracing and immunocytochemical study with special emphasis on calciumbinding proteins. J. Comp. Neurol. 398: 289-307.

11. Geboes, K. and Desmet, V. 1978. Histology of the esophagus. Front. Gastrointest. Res 3: 1-17.

12. Hiura, A. 2000. Neuroanatomical effects of capsaicin on the primary afferent neurons. Arch. Histol. Cytol. 63: 199-215.

13. Holzer, P. 1988. Local effector functions of capsaicin-sensitive sensory nerve endings: involvement of tachykinins, calcitonin gene-related peptide and other neuropeptides. Neuroscience 24: 739-768.

14. Holzer, P. 1991. Capsaicin: cellular targets, mechanisms of action, and selectivity for thin sensory neurons. Pharmacol. Rev. 43: 143-201.

15. Holzer, P. 2004. TRPV1 and the gut: from a tasty receptor for a painful vanilloid to a key player in hyperalgesia. Eur. J. Pharmacol. 500: 231-241.

16. Holzer, P., Schluet, W., Lippe, I.T. and Sametz, W. 1987. Involvement of capsaicin-sensitive sensory neurons in gastrointestinal function. Acta Physiol. Hung. 69: 403-411.

17. Izumi, N., Matsuyama, H., Ko, M., Shimizu, Y. and Takewaki, T. 2003. Role of intrinsic nitrergic neurones on vagally mediated striated muscle contractions in the hamster oesophagus. $J$. Physiol. (Lond.) 551: 287-294.

18. Kerr, K.P., Mitchelson, F. and Coupar, I.M. 1995. Vagal nerve stimulation of the guinea-pig oesophagus. Acta Physiol. Scand. 154: $213-220$
19. Mazzia, C. and Clerc, N. 1997. Ultrastructural relationships of spinal primary afferent fibers with neuronal and non-neuronal cells in the myenteric plexus of the cat esophago-gastric junction. Neuroscience 80: 925-937.

20. Neuhuber, W.L. 1987. Sensory vagal innervation of the rat esophagus and cardia: a light and electron microscopic anterograde tracing study. J. Auton. Nerv. Syst. 20: 243-255.

21. Neuhuber, W.L., Eichhorn, U. and Wörl, J. 2001. Enteric coinnervation of striated muscle fibers in the esophagus: just a "hangover"? Anat. Rec. 262: 41-46.

22. Neuhuber, W.L., Raab, M., Berthoud, H.R. and Wörl, J. 2006. Innervation of the mammalian esophagus. Adv. Anat. Embryol. Cell Biol. 185: 1-73.

23. Numazaki, M. and Tominaga, M. 2004. Nociception and TRP Channels. Curr. Drug Targets CNS Neurol. Disord. 3: 479485.

24. Pal, A. and Brasseur, J.G. 2002. The mechanical advantage of local longitudinal shortening on peristaltic transport. J. Biomech. Eng. 124: 94-100.

25. Rodrigo, J., Hernandez, J., Vidal, M.A. and Pedrosa, J.A. 1975. Vegetative innervation of the esophagus. II. Intraganglionic laminar endings. Acta Anat. 92: 79-100.

26. Shi, G., Pandolfino, J.E., Zhang, Q., Hirano, I., Joehl, R.J. and Kahrilas, P.J. 2003. Deglutitive inhibition affects both esophageal peristaltic amplitude and shortening. Am. J. Physiol. Gastrointest. Liver Physiol. 284: G575-582.

27. Shiina, T., Shimizu, Y., Boudaka, A., Wörl, J. and Takewaki, T. 2006. Tachykinins are involved in local reflex modulation of vagally striated muscle contractions in the rat esophagus via tachykinin $\mathrm{NK}_{1}$ receptors. Neuroscience 139: 495-593.

28. Storr, M., Geisler, F., Neuhuber, W.L., Schusdziarra, V. and Allescher, H.D. 2001. Characterization of vagal input to the rat esophageal muscle. Auton. Neurosci. 91: 1-9.

29. Szolcsányi, J. 2004. Forty years in capsaicin research for sensory pharmacology and physiology. Neuropeptides 38: $377-$ 384.

30. Szolcsányi, J. and Barthó, L. 2001. Capsaicin-sensitive afferents and their role in gastroprotection: an update. J. Physiol. (Paris) 95: 181-188.

31. Tominaga, M. and Caterina, M.J. 2004. Thermosensation and pain. J. Neurobiol. 61: 3-12.

32. Wank, M. and Neuhuber, W.L. 2001. Local differences in vagal afferent innervation of the rat esophagus are reflected by neurochemical differences at the level of the sensory ganglia and by different brainstem projections. J. Comp. Neurol. 435: $41-59$.

33. Ward, S.M., Bayguinov, J., Won, K.J., Grundy, D. and Berthoud, H.R. 2003. Distribution of the vanilloid receptor (VR1) in the gastrointestinal tract. J. Comp. Neurol. 465: 121-135.

34. Wörl, J. and Neuhuber, W.L. 2005. Enteric co-innervation of motor endplates in the esophagus: state of the art ten years after. Histochem. Cell Biol. 123: 117-130. 